\title{
Alteration of the Structure of Human Stratum Corneum Facilitates Transdermal Delivery
}

\author{
Anne Mundstock, Rawad Abdayem, Fabrice Pirot and Marek Haftek ${ }^{*}$
}

Université Lyon 1, EA4169 “Fundamental, clinical and therapeutic aspects of the skin barrier function”; 8 avenue Rockefeller, 69373 Lyon, France

\begin{abstract}
Transdermal transport of pharmacologically active components into the skin depends on the ability of galenic formulations to overcome the stratum corneum (SC) barrier. Microemulsions (ME) are thermodynamically stable liquid systems composed of water, oil and surfactants which may be used for skin permeation and enhance penetration of hydrophobic as well as hydrophilic compounds. We investigated using transmission electron microscopy the effect of ME on human epidermis ex vivo, in order to establish relationship between the type of ME, i.e.: oil-in-water, water-in-oil, gellike, thickened or not with colloidal silica, and the ultrastructural changes in SC barrier resulting from their topical application. ME induced various degrees of dissociation of the SC. The intercellular lipid matrix in the SC became disorganized, which contributed to the separation of corneocytes. This effect was intensified with the increasing oil content in the ME and also when ME were applied under occlusion. The observed morphological changes were in agreement with the increased permeability of ME-treated skin to both lipophilic and hydrophilic compounds reported in the literature. Severe deterioration of the SC barrier induced with the selected ME makes them suitable for selected indications only.
\end{abstract}

Keywords: Epidermal structure, microemulsion, skin barrier, transmission electron microscopy.

\section{INTRODUCTION}

Skin is a very effective barrier which protects the human body from environmental influences such as ultraviolet radiation, chemicals and pathogenic microorganisms. But it also forms a barrier against the loss of water and electrolytes [1]. It is the epidermis that elaborates the cornified layer (stratum corneum, SC) responsible for the skin barrier function [2]. Two parts of the normal SC can be distinguished. The lower part is the SC compactum which is composed of the first 4 to 6 cell layers of the SC [3]. It is this part of the horny layer that provides the effective barrier against water loss. The upper part is called the SC disjunctum and is composed of approximately 8-15 cell layers, more loosely structured, with only lateral cell-cell junctions persisting. The whole SC in human ventral skin consists of around 15-20 cell layers, but these numbers do vary according to the anatomical localization. They are significantly lower on the eyelids and markedly higher on the palms and soles. SC keratinocytes, called corneocytes, are flattened polyedric cells which are filled with keratin and amino acids issued from processing of profilaggrin. Corneocytes possess cornified envelope of cross-linked protein covered with a monolayer of ceramides forming a lipid envelope. The extracellular spaces are filled with lamellar sheets of lipids, primarily cholesterol, free fatty acids and ceramides in roughly equimolar proportions [1].

*Address correspondence to this author at the Research Director with CNRS, Laboratory for Dermatological Research, EA4169 UCBL1, 8, Av. Rockefeller, 69373 Lyon, France; Tel: +33 4787770 30;

Fax:+3347877 75 54; E-mail: marek.haftek@univ-lyon1.fr
These lipid molecules organized in bi-layers provide the relative impermeability of the SC.

The aim of many pharmaceutical formulations is to overcome the epidermal barrier, in order to enable the transport of components into the skin. A former study showed that microemulsions (ME) may have a positive effect on the simultaneous permeation of the antioxidants, like vitamin $\mathrm{C}$ and vitamin $\mathrm{E}$, into the skin [4]. A ME is a system of water, oil and surfactants, which is a transparent, optically isotropic and thermodynamically stable liquid solution. It forms easily, shows low viscosity with Newtonian behavior, displays a high specific surface area and has a very small size distribution. It is possible to dissolve in ME hydrophobic as well as hydrophilic substances. Several potential mechanisms have been described how such formulations can enhance drug permeation through the skin: i) swelling or increased hydration of intracellular keratin caused by denaturation or modification or its conformation; ii) action on desmosomes; iii) alteration of lipid bilayers that may lead to a decreased resistance to permeation; iv) modification of solvent properties of the SC, which changes drug partitioning; v) extraction of lipids from the horny layer with use of particular solvents $[5,6]$. That is why detection of possible ultrastructural changes could be important for the explanation of the functional observations including an increased permeation of the SC. Electron microscopy is a technique which allows observation of such fine structural modifications. In the present study different ME, previously used for transcutaneous delivery of antioxidant vitamins [4], were prepared without any active ingredient. Transmission electron microscopy (TEM) observations of osmium and 
ruthenium tetroxide -stained biopsies were performed in order to explain with ultrastuctural clues the permeabilizing properties of different ME.

\section{MATERIALS AND METHODS}

\section{Preparation of Microemulsions (ME)}

Oil-in-water (o/W) ME, water-in-oil (w/O) ME, gel-like $\mathrm{ME}$ and Aerosil ${ }^{\mathbb{B}}$-thickened ME were all prepared in the same manner using the same set of components (Table $\mathbf{1}$ ). These ME previously showed their efficacy for simultaneous transdermal delivery of both hydrophobic and hydrophilic molecules, vitamins $\mathrm{E}$ and $\mathrm{C}[4,7,8]$. Isopropyl myristate (IPM; Merck, Fleury les Aubrais, France) was used as the lipophilic phase and purified water as the hydrophilic one. The 1:1 mixture of surfactants Tween 40 (polyoxyethylene (20) sorbitan monopalmitate; Fluka Chemie, Buch, Switzerland) and Imwitor 308 (glyceryl caprylate; Condea, Hamburg, Germany) was kindly provided by the Faculty of Pharmacy, University of Ljubljana, Slovenia. The water phases and the oily phases were weighted into the same receptacle. Afterwards the mixture of surfactants was added and the formulations were stirred five minutes with a magnetic stirrer at $400 \mathrm{rpm}$. Gel-like ME was obtained by an increase of the water content at the expense of the lipophilic phase [7]. A thickening agent, colloidal silica (Aerosil ${ }^{\mathbb{B}}$ 200; Degussa, Dusseldorf, Germany) was added to o/w ME to obtain the formulation showing viscosity similar to that of the gel-like ME [8].

\section{Ex Vivo Exposure of Human Skin to ME}

Normal human skin of three female premenopausal donors was obtained from plastic surgery, following the agreement of the local ethics committee at the Civil Hospices of Lyon. The post-operatory fragments of ventral skin were recovered from the operating room, transported in a sterile container at $4^{\circ} \mathrm{C}$, and immediately used for skin permeation experiments, typically within $1 \mathrm{~h}$ after excision.

Each skin fragment was placed on wet tissue impregnated with Dulbecco's modified Eagle medium (DMEM; Gibco, Paris, France) enriched with 5\% fetal calf serum (Gibco), on a heating plate maintained at $32^{\circ} \mathrm{C}$. The permeation experiments were performed in duplicate. Glass chambers were affixed on the skin surface with Super Glue-3 (Loctite). One $\mathrm{mL}$ of each formulation was put into test chambers, two chambers per formulation. Two chambers remained empty, as controls. For each pair of formulations one chamber was left open to simulate non-occlusive conditions while the other chamber was closed with a plug to create occlusion. The control chambers were treated equally. After six hour incubation, the chambers with the applied formulations were carefully removed. Four millimeter punch biopsies were taken from the center of each incubated area and processed for routine histopathology and transmission electron microscopy (TEM).

\section{Transmission Electron Microscopy}

Small skin fragments were fixed with either $2 \%$ glutaraldehyde in sodium cacodylate buffer or $3 \%$ paraformaldehyde in PBS overnight. After washes in appropriate buffer solutions, they were post-fixed with $1 \%$ osmium tetroxide or $0.25 \%$ ruthenium tetroxide, respectively, as previously described [9]. Samples were than dehydrated in graded alcohol and embedded in epoxy resins. Semi-thin and ultra-thin sections were cut perpendicular to

Table 1. Composition of microemulsions used in the present study.

\begin{tabular}{|c|c|c|c|c|c|}
\hline & Gel-like ME & o/W ME & $\begin{array}{c}\text { Thickened }^{*} \\
\text { oW ME }\end{array}$ & w/O ME & $\begin{array}{l}\text { Aerosil }{ }^{\circledR} \\
\text { dispersion }\end{array}$ \\
\hline Water & $60 \%$ & $45 \%$ & $45 \%$ & $10 \%$ & $100 \%$ \\
\hline Isopropyl myristate & $10 \%$ & $25 \%$ & $25 \%$ & $60 \%$ & - \\
\hline $\begin{array}{c}\text { Tween } 40 \\
\text { (polyoxyethylene }(20) \text { sorbitan } \\
\text { monopalmitate) }\end{array}$ & $15 \%$ & $15 \%$ & $15 \%$ & $15 \%$ & - \\
\hline $\begin{array}{c}\text { Imwitor } 308 \\
\text { (glyceryl caprylate) }\end{array}$ & $15 \%$ & $15 \%$ & $15 \%$ & $15 \%$ & - \\
\hline $\begin{array}{l}\text { Aerosil @ } \\
\text { (thickener) }\end{array}$ & - & - & $\begin{array}{l}10 \% \\
\text { in } M E\end{array}$ & - & $\begin{array}{c}10 \% \\
\text { in water }\end{array}$ \\
\hline $\begin{array}{l}o / W=\text { oil in water } \\
W / O=\text { water in oil }\end{array}$ & & * simil & cosities & & \\
\hline
\end{tabular}

*) Thickening agents increase the viscosity of the system without affecting its stability and spontaneous formation. 
the skin surface and counterstained, respectively, with toluidine blue and with uranyl acetate followed by lead citrate. For the samples post-fixed with osmium tetroxide, the magnifications $\times 28,000$ and $\times 75,000$ were used for ultrastructural examination, while the samples post-fixed with ruthenium tetroxide were observed at magnifications of $\times 125,000$ and $\times 260,000$.

\section{RESULTS}

\section{Light Microscopy}

Normal epidermal structure was observed in all the samples. No difference was noticed between the controls with and without occlusion. The Aerosil ${ }^{\circledR}$-dispersion did not induce any notable changes either. The SC was often separated and partially lost in many samples during the preparatory procedures. However, treatment with the w/O ME consistently resulted in the nearly complete elimination of the horny cells, especially under occlusion, and the o/W ME induced a notable relaxation of the SC (Fig. 1).

\section{TEM of ME-Treated Human Skin Explants}

Standard post-fixation with osmium tetroxide allowed observation of the tissue morphology, except of the fine structure of intercellular lipid lamellae in the SC $[10,11]$. When compared to the untreated control (Fig. 2a), there was no major change in the SC morphology after application of the Aerosil ${ }^{\circledR}$-dispersion alone, without lipophilic phase or surfactants (Fig. 2b), (mean number of layers in the SC compactum $+/-\mathrm{SD}=5.67+/-0.52)$. Also the gel-like $\mathrm{ME}$, with a relatively low contents of isopropyl myristate, induced only minor ultrastructural defects in the SC, such as discrete swelling of the intercellular spaces and the beginning of detachment in the SC disjunctum (mean number of layers in the SC compactum $+/-\mathrm{SD}=5.33+/-$ 0.82 ; Fig. 3). In contrast, the treatment with the o/W ME resulted in an extensive dissociation of the $\mathrm{SC}$ disjunctum and a correlated reduction of the $\mathrm{SC}$ compactum (mean number of layers in the SC compactum $+/-\mathrm{SD}=3.67+/$ 0.52 ; Fig. 4). Additionally, amorphous and composite intercorneocyte inclusions were observed in the upper part of the cohesive SC (Fig. 4b). The strongest effect was exerted by the w/O ME, application of which led to the pronounced dissociation between the SC cells. Typically, there were only a couple of corneocytes remaining at the surface of the living epidermis (mean number of layers in the SC compactum $+/$ $\mathrm{SD}=1.83+/-0.75$; Fig. 5). Occlusion, in general, amplified the degree of the observed ultrastructural changes whereas addition of the thickener had a somewhat attenuating effect (Fig. 6). Ultrastructural changes were, thus, induced by all tested ME formulations and, with the constant proportion of surfactants, the extent of these modifications paralleled the increasing proportion of the lipophilic phase.

After ruthenium tetroxide staining, the controls showed intercellular spaces filled with the lipid lamellae in the SC compactum (Fig. 7a). In the gel-like ME -treated samples, the deeper layers of the SC remained attached to the living epidermis and the intercorneocyte spaces were filled with the well-structured lipid lamellae (Fig. 3b). A nearly complete

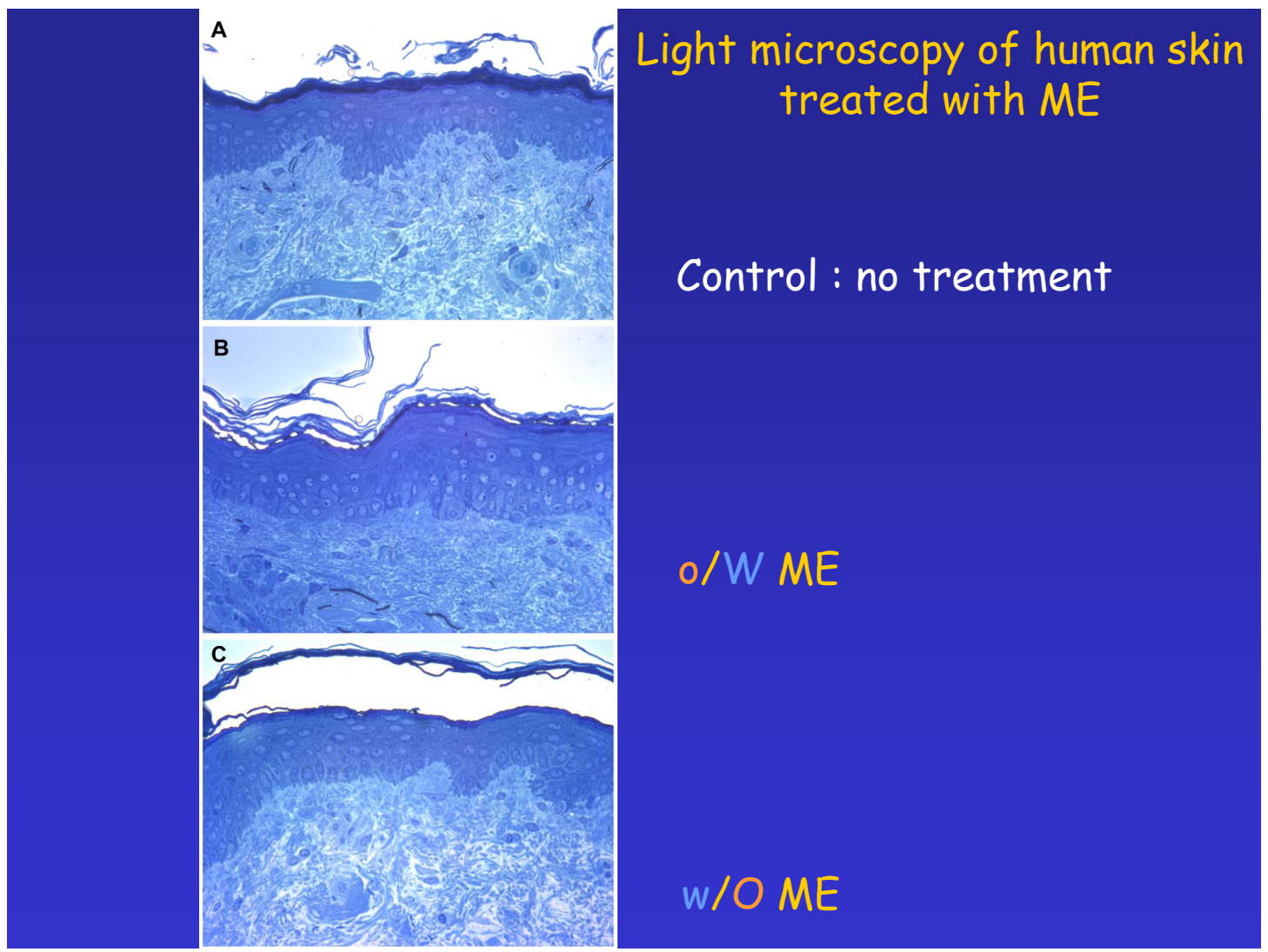

Fig. (1). Semi-thin sections observed at the light microscopy level do not show remarkable histological changes imputable to ME application, as compared to the non-treated controls (A), except of the SC relaxation (B: oil in water ME) and frequent loss of the upper SC layers in samples treated with the water in oil ME (C). Toluidine blue staining. Magnification: x400. 


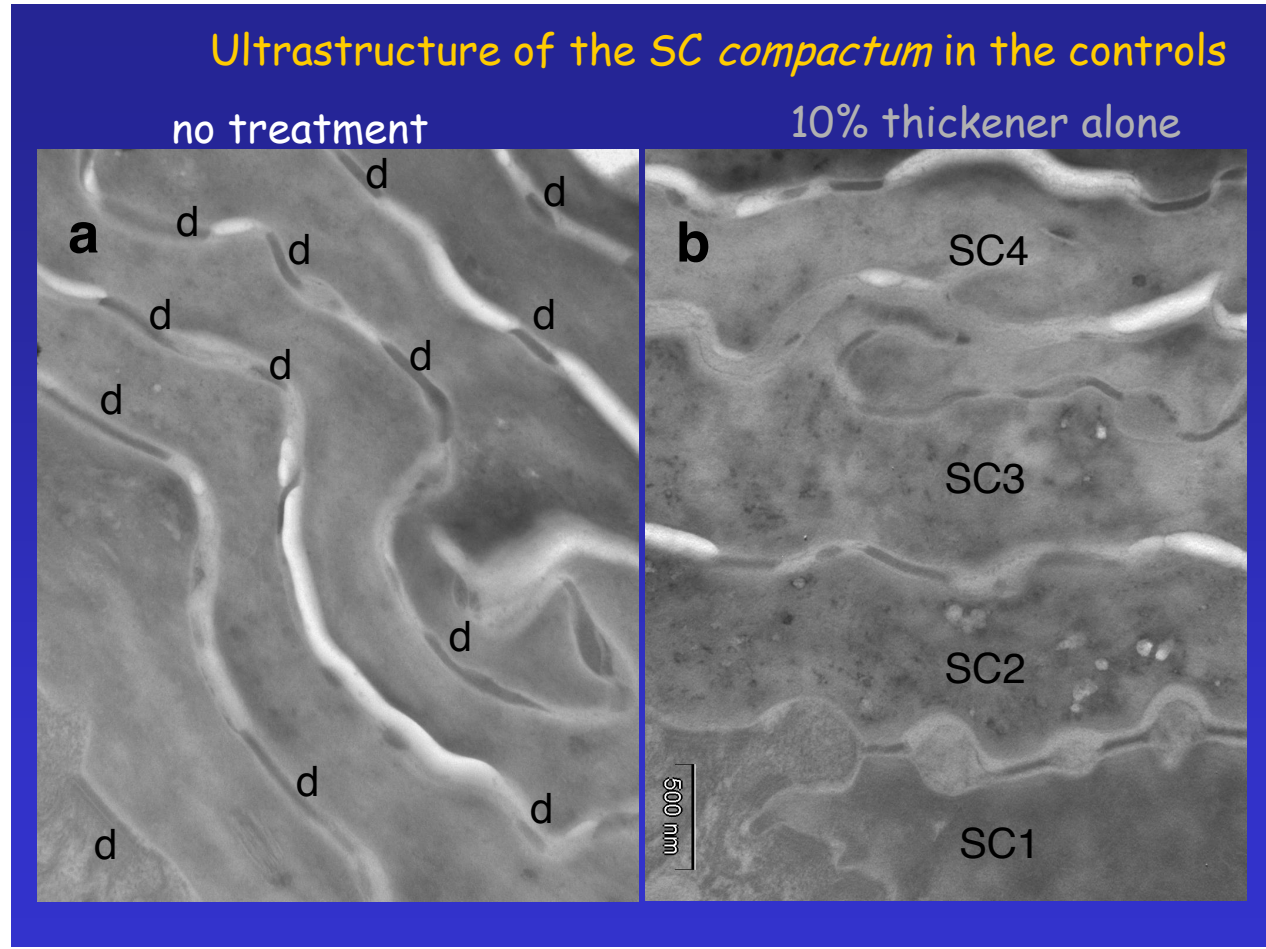

Fig. (2). TEM of the control SC. a) no treatment, normal SC compactum; b) no major influence due to the application of the thickener $\left(\right.$ Aerosil $\left.^{\mathbb{B}}\right)$. d, corneodesmosomes in (a); SC 1-4, the first four SC layers.

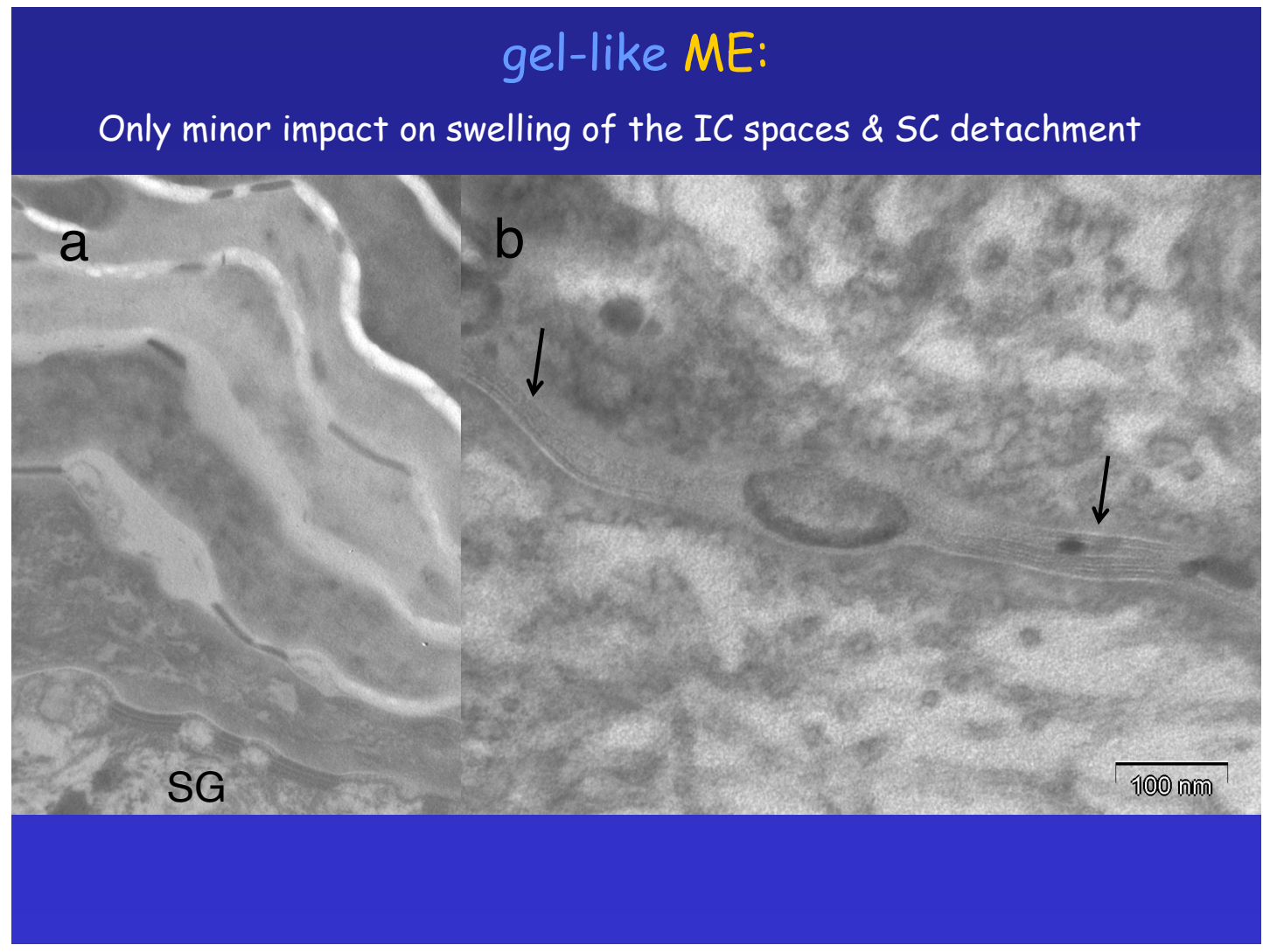

Fig. (3). TEM of the SC after application of the gel-like ME. a) Only minor impact on swelling of the intercellular spaces and SC detachment

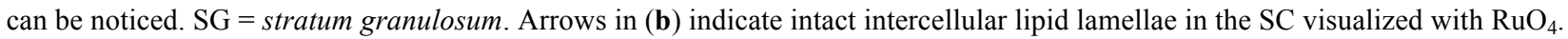

separation of the SC could be confirmed after incubation with w/O ME. Only the lipid envelopes stayed attached at the surface of the corneocytes, whereas loose intercellular lipid lamellae could be sometimes distinguished within the otherwise unstructured or altogether empty intercorneocyte spaces (Fig. 7b). 


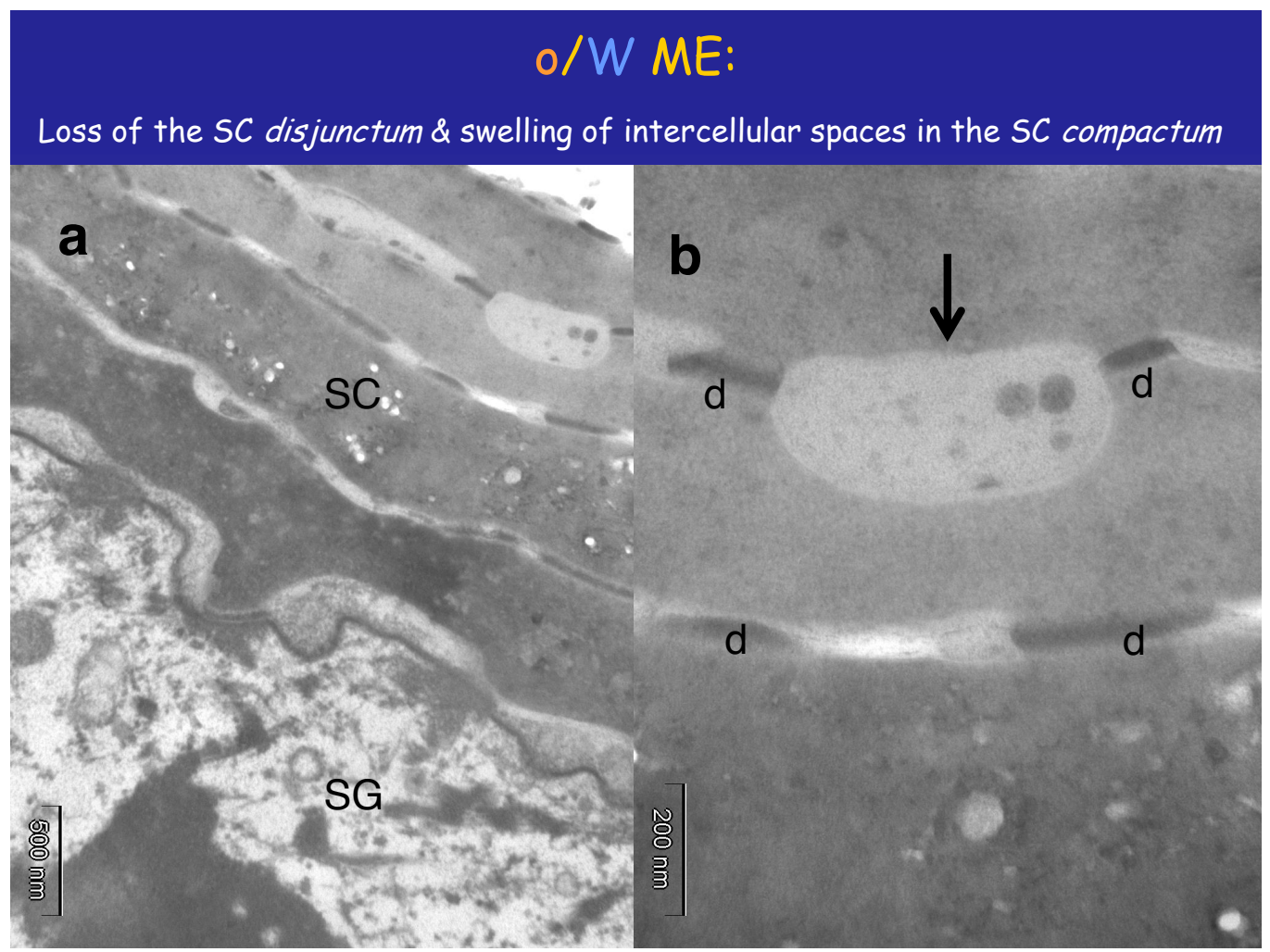

Fig. (4). Treatment of the SC with the oil in water ME provokes a partial loss of the SC disjunctum and swelling of intercellular spaces in the SC compactum. Here, only 4 corneocytes are left (a) and intercellular amorphous and composite inclusions are visible (arrow in b). d, corneodesmosomes. $\mathrm{SG}=$ stratum granulosum $; \mathrm{SC}=$ stratum corneum .

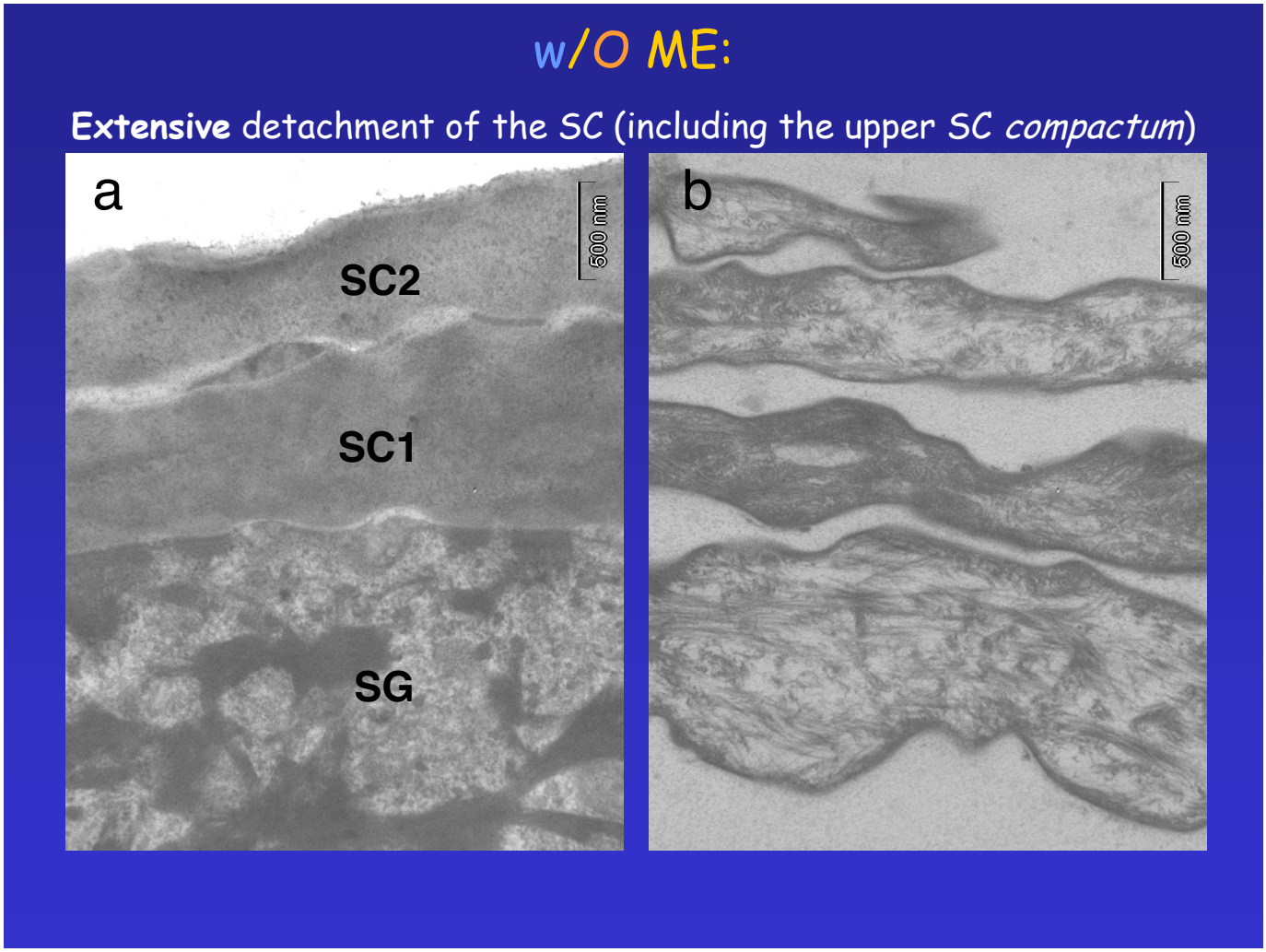

Fig. (5). Extensive detachment of the SC is observed after application of the water in oil ME. a) two corneocytes of SC compactum are left over the SG keratinocyte; b) loose corneocytes detaching from the sample in the upper part of the SC entirely separated from the remaining tissue; no corneodesmosomes. 


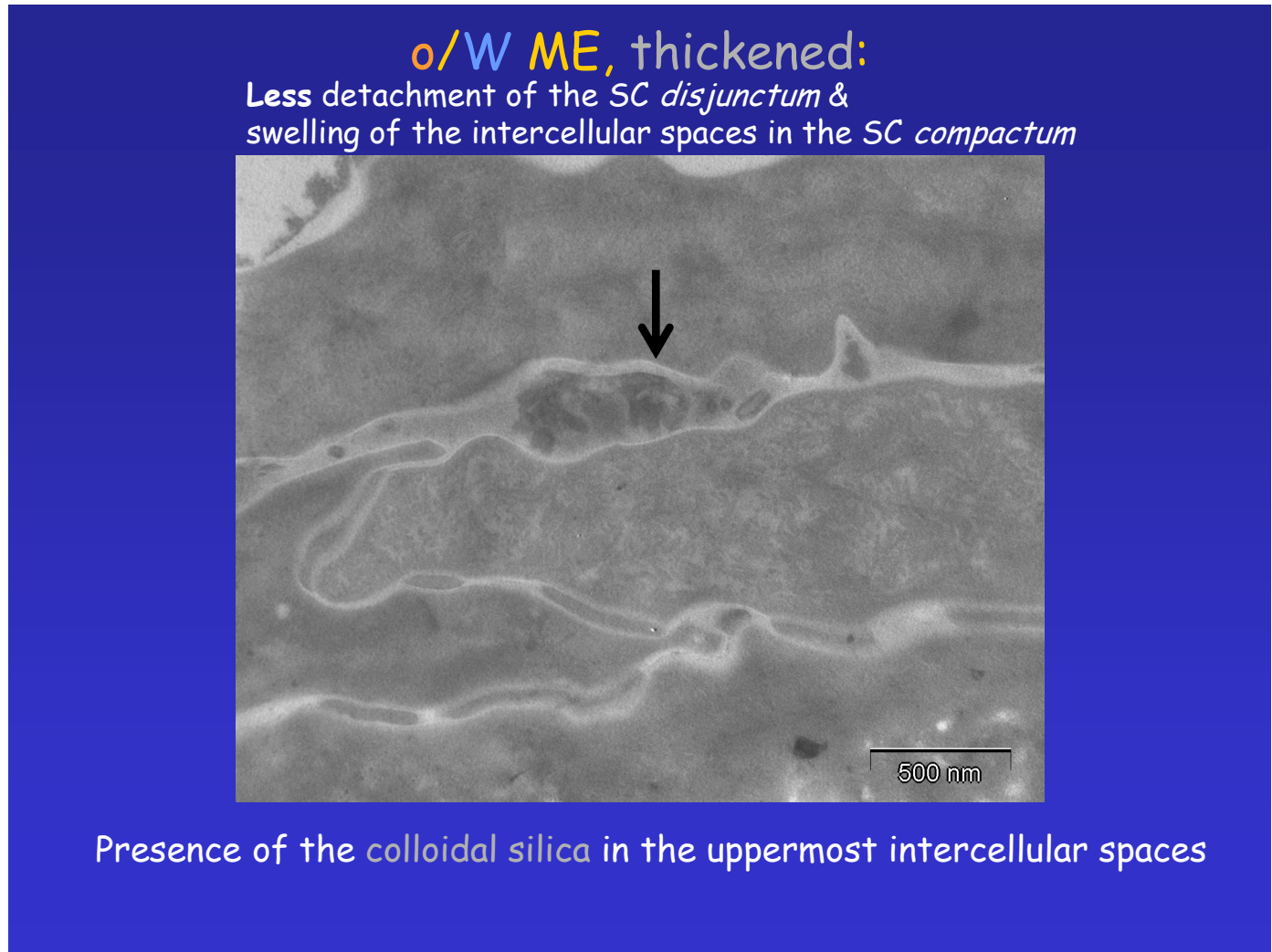

Fig. (6). Addition of thickener to the oil in water ME results in less detachment of the SC disjunctum. Intercellular deposits of the thickener are visualized in the upper SC (arrow).

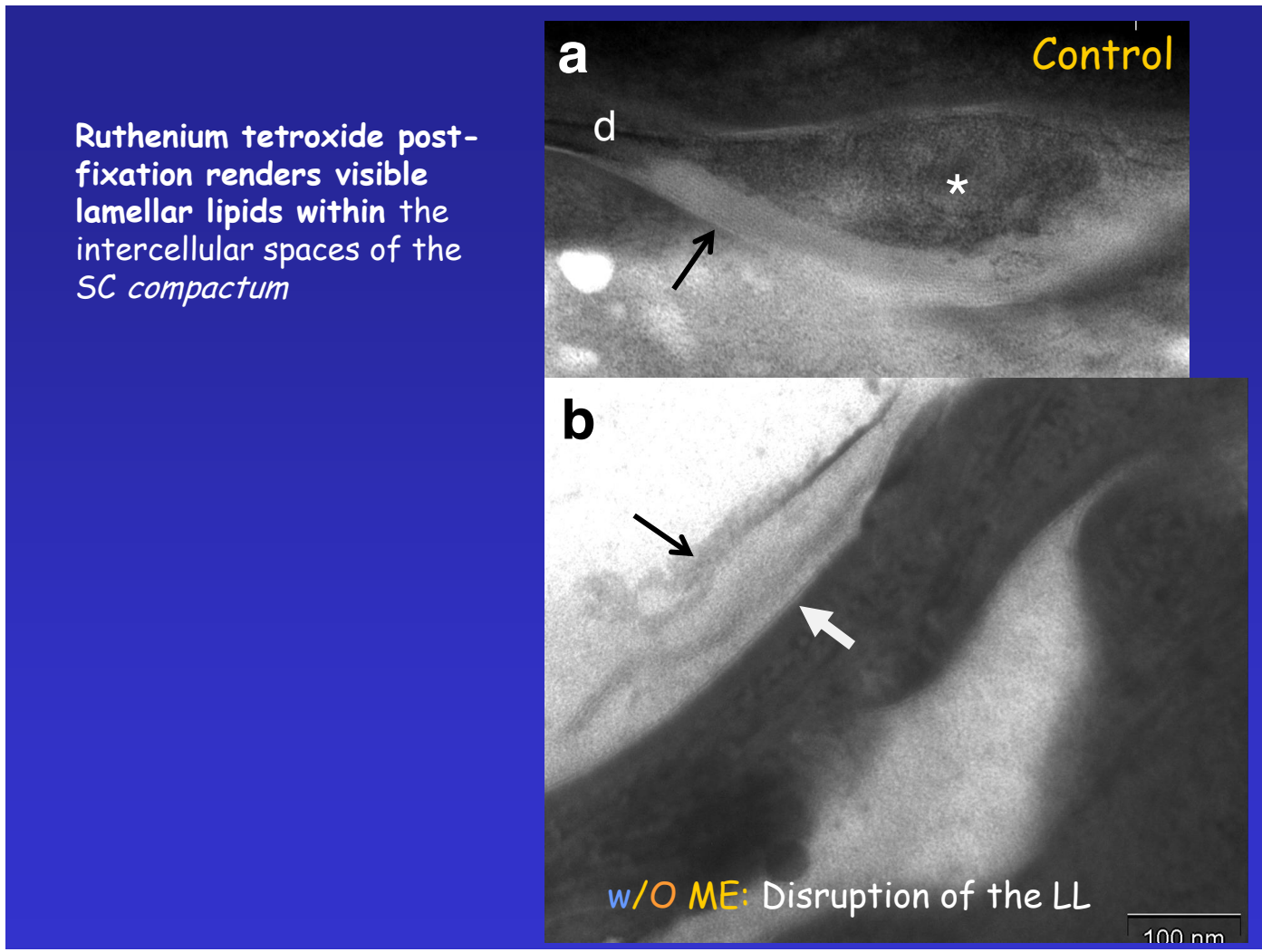

Fig. (7). Ruthenium tetroxide staining of the SC. a) intact lamellar lipids (LL) in the SC compactum of a control sample (arrow). b) Water/oil ME causes total disruption of the intercellular lipids. Residual lamellae are indicated with the black arrow. Thick white arrow points to the corneocyte lipid envelope. Corneodesmosome $=\mathrm{d}$; Intercellular hydrophilic lacuna $=$ asterisk. 


\section{DISCUSSION}

It could be clearly demonstrated that the changes in morphology of the SC were dependent on the proportions between ingredients used for ME formulation. Specifically, the growing impact of ME on the SC overall structure and lamellar lipid disruption correlated with the increasing oil content: gel-like $<\mathrm{o} / \mathrm{W}<\mathrm{w} / \mathrm{O}$. Indeed, it has been previously observed using attenuated total reflectance Fourier transform infrared spectroscopy (ATR-FTIR) on pig ear substrates that $\mathrm{ME}$-induced changes to the SC appear to be proportional to the level of uptake of individual ME components [12]. Consequently, isopropyl myristate and surfactant - cosurfactant mix used in our study, which segregate to the lipophilic compartment, could be predicted to disturb the intercellular lamellar lipids in a dose-dependent manner. Ruthenium tetroxide staining, which enabled us to observe the organization of the lipid lamellae in the spaces between the corneocytes, confirmed that the most profound changes in this compartment occurred after incubation with the w/O ME. Because intercellular lamellar lipids are considered essential for the skin barrier function $[1,13]$, their disruption after application of ME with high lipophilic content should induce an increase in skin permeability. Indeed, it was found that ME of the same type as described in this study disturbed skin barrier function [4]. This was revealed by an increased transepidermal water loss (TEWL), a well-established method to determine integrity of the skin barrier [14]. However, in that earlier study [4], it was the gel-like formulation that showed the most pronounced « irritation potential » ex vivo (TEWL measurements on pig ear skin) and toxicity in vitro (MTT on reconstructed human epidermis). This indicates that despite of being a potentially interesting tool for prediction of skin penetration and tolerance, the ultrastructural examination of ex vivo treated human skin may yield results varying from the other tests, most probably depending on the different experimental settings.

In addition to the oil content, another possible reason for the observed dissociation of the SC could be the influence of surfactants used in the ME formulations. Apart from their impact on the extracellular lipids, surfactants can also act on proteins and possibly facilitate degradation of the protein structures [3], such as cell-cell junctions in the SC, i.e. corneodesmosomes. Nevertheless, in the present study, the proportions of surfactants in different ME were kept constant, so it is unlikely that this latter mechanism of action was primarily responsible for the observed differences in the degree of SC dissociation in the treated samples. The observed relaxation of the SC structure after application of $\mathrm{ME}$ and the resulting partial loss of the permeability barrier could contribute to the explanation of the fact that ME, even those with a low oil content, were able to increase simultaneously penetration of both hydrophilic and hydrophobic drugs $[4,6,15]$.

Under our experimental conditions, no significant morphological alteration of the living epidermal layers was observed after 6 hour application of ME. This remains in agreement with the previous reports dealing with the same kinds of formulations tested in vitro on reconstructed human epidermis [4]. Nevertheless, based on this morphological study, our prediction is that with the constant proportion of surfactants, the permeation efficacy of ME should increase along with the proportion of the lipophilic fraction. The ransom of this effect could be the potential adverse effects. One has to keep in mind that a reduced barrier function facilitates penetration of various chemicals through the slightly damaged skin [16] and this represents a potential risk and limitation to the use of most "aggressive" formulations.

\section{AUTHORS' CONTRIBUTIONS}

All authors provided substantial contributions to the conception and design, acquisition of data, or analysis and interpretation of data, to the drafting of the article or revising it critically for important intellectual content.

\section{ABBREVIATIONS}
$\mathrm{ME}=$ Microemulsion
$\mathrm{o} / \mathrm{W}=$ Oil in water
$\mathrm{SC}=$ Stratum corneum
$\mathrm{SG}=$ Stratum granulosum
$\mathrm{TEM}=$ Transmission electron microscopy
$\mathrm{w} / \mathrm{O}=$ Water in oil

\section{CONFLICT OF INTEREST}

The authors confirm that this article content has no conflict of interest.

\section{ACKNOWLEDGMENTS}

The authors would like to thank Prof. Dr. Alfred Fahr and Prof. Françoise Falson for making possible student (AM) exchange between Friedrich-Schiller-Universität Jena, Germany and the Université Claude Bernard Lyon 1, France. The ultrastructural observations were performed at the Centre Technique des Microstructures (CT $\mu$ ) belonging to the Lyon Bio Image platform of University Lyon 1.

The study was supported by COST action SKINBAD (BM0903).

\section{REFERENCES}

[1] Feingold KR, Elias PM. Role of lipids in the formation and maintenance of the cutaneous permeability barrier. Biochim Biophys Acta 2014; 1841: 280-94.

[2] Haftek M. 'Memory' of the stratum corneum: exploration of the epidermis' past. Br J Dermatol 2014; 171(Suppl 3): 6-9.

[3] Fartasch M. Ultrastructure of the epidermal barrier after irritation. Microsc Res Tech 1997; 37: 193-9.

[4] Rozman B, Gasperlin M, Tinois-Tessoneaud E, Pirot F, Falson F. Simultaneous absorption of vitamins $\mathrm{C}$ and $\mathrm{E}$ from topical microemulsions using reconstructed human epidermis as a skin model. Eur J Pharm Biopharm 2009; 72: 69-75.

[5] Kogan A, Garti N. Microemulsions as transdermal drug delivery vehicles. Adv Colloid Interface Sci 2006; 123-126: 369-85.

[6] Kreilgaard M. Influence of microemulsions on cutaneous drug delivery. Adv Drug Deliv Rev 2002; 54(Suppl 1): 77-98.

[7] Rozman B, Zvonar A, Falson F, Gasperlin M. Temperaturesensitive microemulsion gel: an effective topical delivery system for simultaneous delivery of vitamins $\mathrm{C}$ and $\mathrm{E}$. AAPS PharmSciTech 2009; 10: 54-61.

[8] Rozman B, Gosenca M, Gasperlin M, Padois K, Falson F. Dual influence of colloidal silica on skin deposition of vitamins $\mathrm{C}$ and $\mathrm{E}$ simultaneously incorporated in topical microemulsions. Drug Dev Ind Pharm 2010; 36: 852-60. 
[9] Haftek M, Teillon MH, Schmitt D. Stratum corneum, corneodesmosomes and ex vivo percutaneous penetration. Microsc Res Techn 1998; 43: 224-49.

[10] Schwartzendruber DC, Burnett IH, Wertz PW, Madison KC, Squier CA. Osmium tetroxide and ruthenium tetroxide are complementary reagents for the preparation of epidermal samples for transmission electron microscopy. J Invest Dermatol 1995; 104: 417-20.

[11] Fartasch M, Bassukas ID, Diepgen TL. Structural relationship between epidermal lipid lamellae, lamellar bodies and desmosomes in human epidermis: an ultrastructural study. Br J Dermatol 1993; 128: 1-9.

[12] Hathout RM, Mansour S, Mortada ND, Geneidi AS, Guy RH. Uptake of microemulsion components into the stratum corneum and their molecular effects on skin barrier function. Mol Pharm 2010; $7: 1266-73$
[13] Boddé HE, Kruithof MAM, Brussee J, Koerten HK. Visualisation of normal and enhanced $\mathrm{HgCl}_{2}$ transport through human skin in vitro. Int J Pharm 1989; 53: 13-24.

[14] Netzlaff F, Kostka KH, Lehr CM, Schaefer UF. TEWL measurement as a routine method for evaluation the integrity of epidermis sheets in static Franz type diffusion cells in vitro. Limitations shown by transport data testing, Europ J Pharm Biopharm 2006; 63: 44-50.

[15] Lopes LB. Overcoming the cutaneous barrier with microemulsions. Pharmaceutics 2014; 6: 52-77.

[16] Nielsen JB, Nielsen F, Sorensen JA. Defence against dermal exposure is only skin deep: significantly increased penetration through slightly damaged skin. Arch Dermatol Res 2007; 299: 42331.

(C) Mundstock et al.; Licensee Bentham Open.

This is an open access article licensed under the terms of the Creative Commons Attribution Non-Commercial License (http://creativecommons.org/licenses/by-nc/3.0/) which permits unrestricted, non-commercial use, distribution and reproduction in any medium, provided the work is properly cited. 